\title{
MODELO DIDÁCTICO MEDIACIONAL PARA PROMOVER EL APRENDIZAJE DE SEGUNDAS LENGUAS
}

\author{
(DIDACTIC AND MEDIATING MODEL TO PROMOTE SECOND LANGUAGE LEARNING)
}

Jorge Landaverde y Trejo

Universidad Autónoma de Querétaro (México)

\section{RESUMEN}

La población mexicana presenta una tendencia a crecer menos y a envejecer más, lo cual se refleja en un decrecimiento de la población en edad escolar y el incremento de la población en edad laboral. Esto exige nuevos enfoques educativos que aseguren la formación para la vida. El modelo didáctico mediacional está pensado para contribuir a la transición de un modelo de educación escolarizada, al modelo de educación mixta y desescolarizada, para satisfacer una demanda que está transitando de la escuela al ámbito laboral. Dentro del aprendizaje para la vida juega un papel muy importante la apertura a otras culturas. Es aquí donde consideramos necesaria la promoción del aprendizaje de segundas lenguas mediante un modelo didáctico que haga uso de todas las mediaciones a nuestro alcance, especialmente diseñando contenidos digitales que promuevan la intercomunicación cultural teniendo como eje central la promoción del plurilingualismo para la integración social.

\begin{abstract}
The Mexican population presents a trend to grow less and to get older, which is reflected in a decrease of the population of school age and an increase in the population of work age. This requires new educational approaches to ensure the formation through life. The didactic mediating model is thought to contribute to the transition of a scholar educational model, into the blended and informal education, to satisfy a demand which is moving from the school to the labor context. Within the learning through life the openness to other cultures plays an important role. It's here where we consider necessary the promotion of second languages learning via a didactic model which makes usage of all the mediations at hand, especially by designing digital contents which promote the cultural intercommunication having as a central axis the promotion of the pluriculturalism for the social integration.
\end{abstract}


JORGE LANDAVERDE Y TREJO

MODELO DIDÁCTICO MEDIACIONAL PARA PROMOVER EL APRENDIZAJE DE SEGUNDAS LENGUAS

\section{Relevancia del tema}

\section{a) Justificación}

En el contexto mexicano el grueso de la población tiende a concentrarse entre generaciones de jóvenes y adultos de los cuales, una gran mayoría, ha dejado de asistir a procesos educativos formales (Cfr. CENEVAL, 2005). Por su parte, el factor denominado "mundialización" incrementa la necesidad de apertura a otras culturas $y$, por ende, a otras lenguas para lograr mayor eficiencia laboral y acceder a estilos de vida más satisfactorios.

De entre los resultados de un análisis de necesidades en torno al diseño y producción de contenidos digitales para promover el aprendizaje de lenguas se destacan los siguientes datos: del total de ponencias incluidas en la Memoria del último Foro de Especialistas Universitarios en Lenguas Extranjeras1, se observa que únicamente el $2 \%$ de las ponencias hace referencia directa a la Capacitación de Adultos y escasamente el $12 \%$ de dichas ponencias versa sobre diseño y producción de materiales didácticos para el aprendizaje de lenguas con apoyo en los medios tecnológicos.

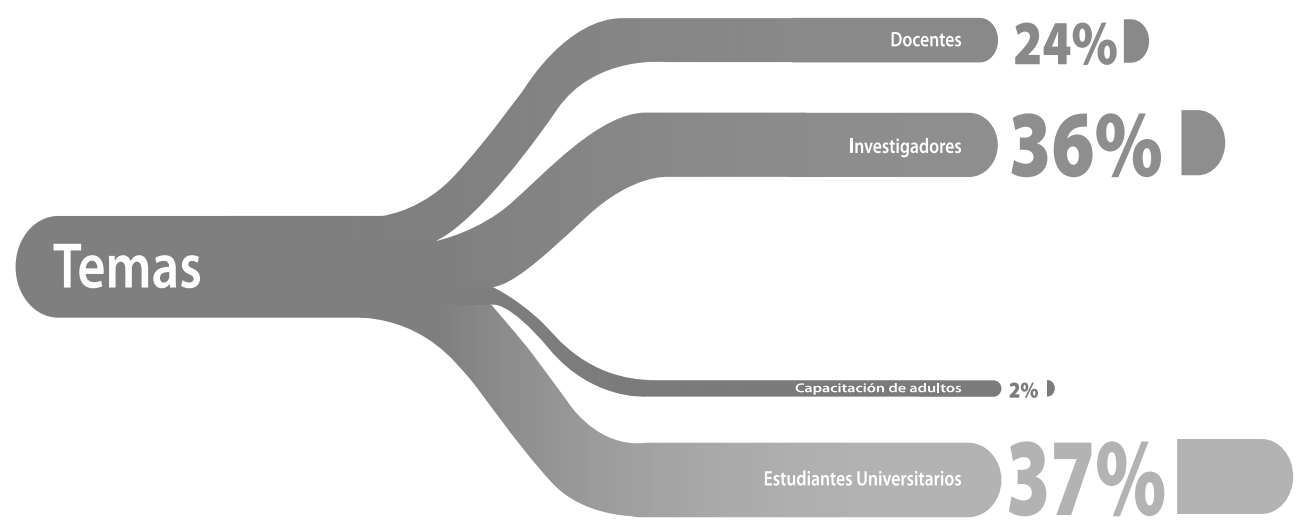

De lo arriba señalado se infiere que hay un amplio sector poblacional que demanda una capacitación fuera del aula. De ahí que para superar las barreras de sincronía y escasez de recursos económicos, se propone el diseño y producción de contenidos digitales en formato CD con la intención de promover el aprendizaje de lenguas entre personas que no pueden dedicar tiempo completo a su instrucción formal, sea porque tienen una carga laboral que les impide acceder a una educación 
JORGE LANDAVERDE Y TREJO

MODELO DIDÁCTICO MEDIACIONAL PARA PROMOVER EL APRENDIZAJE DE SEGUNDAS LENGUAS

formal presencial, o bien, por cualquier otro impedimento de índole diversa como lo es la limitante económica.

\section{b) Categorías referenciales}

\section{Didactización}

Como docente de una universidad pública en México, mi quehacer cotidiano ha caído dentro de la educación formal. Sin embargo, con cierta frecuencia me encuentran ex-alumnos de la materia de inglés que imparto y me comentan que están de regreso de los Estados Unidos o Canadá. Como inmigrantes en dichos países, han experimentado la necesidad de saber cómo comunicarse en inglés, y externan un fuerte deseo por seguir aprendiendo el idioma; sin embargo, arguyen no poder asistir a cursos presenciales debido a su carga laboral. Requieren de una modalidad abierta.

En una encuesta aplicada a dos grupos de aprendientes de inglés como lengua extranjera cuyos participantes tenían edades promedio de 33.38 y 41.05 años, respectivamente, se detectó que, según la opinión de los encuestados, el principal factor que obstaculiza el aprendizaje de inglés como lengua extranjera es la 'falta de tiempo'. Dicha expresión 'falta de tiempo', en entrevistas personales, se ha detectado como sinónimo de falta de: interés, motivación, estrategias adecuadas, entornos favorables y presentación adecuada de materiales y contenidos de aprendizaje.

En atención a la detectada necesidad por acceder a un aprendizaje de inglés a través de materiales motivadores y disponibles para procesos de autoaprendizaje nos parecen esenciales las dos premisas de la didactización que propone el autor Rolf Arnold (2005) 2 : la primera establece que no es necesario promover el aprendizaje de todo lo que se puede decir sobre el tema por lo que es preciso eliminar contenidos superfluos para lograr mayor accesibilidad; la segunda premisa indica que lo relevante no es el conocimiento en sí, sino el desarrollo de estrategias de exploración y aplicación de tal conocimiento. Por lo cual es preciso diseñar medidas didácticas que favorezcan el descubrimiento y la acción transformadora.

\section{Mediación}

En el debate que se ha venido dando entre lo que son las competencias laborales versus las competencias académicas, aparece una tercera cuestión que es la de las competencias mediacionales. 
JORGE LANDAVERDE Y TREJO

MODELO DIDÁCTICO MEDIACIONAL PARA PROMOVER EL APRENDIZAJE DE SEGUNDAS LENGUAS

Como una primera aproximación al debate entre las competencias laborales y las competencias académicas se muestra un esquema (Ver anexo 1) basado en Barrón (2002) 3 el cual permite deslindar lo concerniente al ámbito académico cuya racionalidad se funda en las necesidades básicas de aprendizaje con reconocimiento de la dimensión histórico social y la identidad cultural del aprendiente, respecto de las cambiantes perspectivas del campo laboral en donde, para evitar la unilateralidad ejercida por el sector empresarial se debe impulsar la integración de consejos formados por representantes de los diferentes sectores sociales, tales como los gubernamentales, educativos; así como los empresariales y sindicales, a fin de converger en políticas gubernamentales que regulen satisfactoriamente el mercado de trabajo.

Como un segundo acercamiento al tema de las competencias, resulta pertinente citar el modelo mediacional que propone la autora (Chan, 2004) 4 quien plantea la necesidad de explicitar cada uno de los ingredientes que la nueva era tecnológica está requiriendo: hay la tendencia a dar por supuesto el desarrollo de competencias mediacionales para un uso adecuado de las Tecnologías de la Información y la Comunicación sin considerar sus implicaciones en términos de formación docente e inducción discente. Dicha autora presenta su modelo heurístico mediacional destacando su intencionalidad de "vincular la producción y uso de conocimiento al diseño educativo y a la virtualidad desde un paradigma comunicacional." (Chan, 2004, p. 33). Dicha autora presenta en su libro un cuadro que denomina: Seis miradas sobre el concepto de mediación. En dicho cuadro se resume que la mirada de Manuel Martín Serrano (1994) reconoce las mediaciones socioculturales y las de orden lógico, pero desconoce las mediaciones tecnológicas. La mirada de Guillermo Orozco (1990) considera las mediaciones como procesos de negociación de significados y las tipifica en cognoscitivas, culturales, de referencia, institucionales y video-tecnológicas. De Gutiérrez y Prieto (1991) observa tres tratamientos de los materiales educativos incluidos en la mediación pedagógica: 1) desde el tema: ubicación, temática, contenidos, estrategias de lenguaje, conceptos; 2) desde el aprendizaje: el interlocutor presente, autoaprendizaje, el juego pedagógico; 3) tratamiento formal: enriquecimiento de la percepción y la textualidad. Por su parte, José Manuel Pérez Tornero considera la mediación como soporte mediático de los actos de comunicación: proceso por el que se brinda soporte, con medios, para registrar, representar y trasladar signos y lenguaje preexistentes. Transformación de los actos de comunicación que antes tenían una naturaleza física precisa y que ahora se tornan en virtualidad. 
JORGE LANDAVERDE Y TREJO

MODELO DIDÁCTICO MEDIACIONAL PARA PROMOVER EL APRENDIZAJE DE SEGUNDAS LENGUAS

\section{Secuenciación en el aprendizaje de lenguas}

En los procesos cognoscitivos que se dan en el aprendizaje de segundas lenguas diversos autores han rastreado y descrito una secuencia que parte de una primera etapa de adquisición de vocabulario, seguida de la adquisición de fórmulas sintácticas, las cuales son enriquecidas con una reestructuración semántica cada vez más compleja 5. Es lo que se ha dado en llamar: Lexicalización-SintaxizaciónRelexicalización. El autor Skehan cuando comenta sobre las representaciones mentales del lenguaje hace referencia a dos sistemas combinados: uno basado en reglas; otro basado en ejemplos o modelos, los cuales funcionan combinados en un sistema de manera dual. Cuando es dominante el sistema basado en modelos se da un acercamiento a la imitación asegurando fluidez; cuando predomina el sistema basado en reglas se da mayor creatividad a expensas de menor fluidez. Las etapas iniciales de la adquisición de la lengua materna son primordialmente léxicas. En etapas posteriores se dan procesos de sintaxización operando sobre el repertorio léxico que el niño ha desarrollado. Como resultado de lo anterior, esa lengua basada en léxico se reorganiza para llegar a ser basada en sintaxis y entonces aparece un sistema gobernado por reglas. Posteriormente se vuelven a analizar las partes léxicas y se reestructuran unidades más complejas de palabras identificadas por su función sintáctica y comunicativa (relexicalización).

Por otra parte, habrá de considerarse que en el aprendizaje de segundas lenguas no aplica el principio lógico-matemático que establece que "el orden de los factores no altera el producto". En los procesos de adquisición y aprendizaje de lenguas existen secuencias que avanzan de menor a mayor complejidad cuyo orden es preciso respetar si se pretende lograr precisión y fluidez. En los procesos mentales que van de las emisiones de textos (sonoros, escritos y/o icónicos) que llegan como un todo (input) al destinatario, la recepción es parcial (intake) 6 por intermediación de un sistema de interlenguaje en construcción; tales procesos requieren de una práctica controlada y un tiempo razonable antes de que el aprendiente o receptor esté en posibilidades de emitir una producción creativa y transformadora.

\section{c) Modelo Didáctico Mediacional}

A partir de una aproximación a la comprensión de los procesos inherentes a la adquisición/aprendizaje de segundas lenguas 7 , se han venido diseñando y produciendo contenidos digitales para promover el aprendizaje de inglés como lengua extranjera, a través de entornos mixtos de aprendizaje. 
JORGE LANDAVERDE Y TREJO

MODELO DIDÁCTICO MEDIACIONAL PARA PROMOVER EL APRENDIZAJE DE SEGUNDAS LENGUAS

\section{Diagrama de los procesos mentales subyacentes al lenguaje}

En el Anexo 2 se advierte la complejidad 8 de los procesos mentales inherentes a los procesos cognitivos implicados en el lenguaje. El diagrama muestra los bucles retroactivos y recursivos que se dan en las interacciones comunicativas que implican los actos lingüísticos. Recurriendo al concepto complejo de sistema se propone un esquema denominado: Sistemas Interrelacionados que promueven el Plurilingualismo. En este esquema se visualiza una reciprocidad circular entre la tríada: sistema, organización e interrelación. La interrelación como mediación entre sistemas y organización. Organización como producto de las acciones y relaciones entre emisores y receptores. Aquí la retroacción rompe la causalidad lineal ya que el efecto repercute en la causa y la modifica haciendo posible salir de un cerramiento del sistema hacia bucles recursivos donde el efecto último pasa a ser el elemento primero y la primera causa de nuevos bucles retroactivos y recursivos.

\section{Excentricidad del Modelo Didáctico Mediacional}

Después de habernos percatado de la complejidad de los actos comunicativolingüísticos es preciso deslindar las funciones que corresponden a cada uno de los actores implicados en el aprendizaje de segundas lenguas. Aún cuando la adquisición y el aprendizaje de lenguas tienen una fuerte carga subjetiva e individual, lo cual sugiere perspectivas de autoaprendizaje, sin embargo, las representaciones mentales subjetivas tienen un referente social ineludible y, en el caso del desarrollo de competencias comunicativo-lingüísticas, el aspecto sociocultural subyace a cada uno de los elementos desde la emisión, el emisor, las interfaces, la recepción y el receptor, así como todos los procesos mentales implicados.

En ese contexto, el modelo tradicional del docente como centro o único protagonista de la enseñanza resulta totalmente obsoleto. Incluso el aprendizaje centrado en el aprendiente no basta para enfrentar la problemática de complejidad implicada en esta era mediática. Tampoco la sustitución de lo artesanal por los avances tecnológicos y mediáticos asegura de manera mágica el acceso a un aprendizaje de lenguas en un contexto mediatizado de mundos icónicos, simbólicos y virtuales.

El contexto mundializado-mediatizado actual clama por el desarrollo de nuevas competencias laborales, académicas y mediacionales. Pero ¿desde qué perspectiva teórica? 
JoRge LANDAVERDE Y TREJO

MODELO DIDÁCTICO MEDIACIONAL PARA PROMOVER EL APRENDIZAJE DE SEGUNDAS LENGUAS

A partir de una encuesta aplicada a un grupo de aprendientes en un curso de inglés con modalidad semipresencial en la cual se evaluó con más alto puntaje a ciertos materiales de autoaprendizaje, por encima de los docentes, de los asesores y de los mismos aprendientes, el papel protagónico del enseñante y de los aprendientes quedó como al desnudo y el paradigma del aprendizaje centrado en el aprendiente fue cuestionado 9. Entonces cobró sentido la propuesta del Dr. Enrique RuizVelasco Sánchez (2005) de un Modelo Educativo Excéntrico quien sugiere que "en lugar de cursos magistrales impartidos por el profesor a los alumnos, se propicie la descentralización y excentricidad dela enseñanza, asegurando asíla continuidad entre los grupos de educandos excéntricos. (Ruiz-Velasco, 2005, citado en Landaverde, 2006, p.117). El modelo se denomina excéntrico no por su extravagancia sino por los movimientos alternos que son producidos por una palanca excéntrica posicionada en un eje de giro adicional al eje de rotación central como lo muestra el siguiente dibujo:

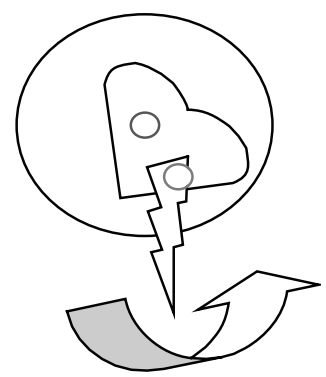

\section{Resultados obtenidos}

\section{a) Pasos dados}

Se han dado pasos en el diseñoy producción de contenidos didácticos digitalizados de los cuales se destacan las siguientes muestras.

- En el SOMECE de 2003 (XIX Simposio Internacional de Computación en la Educación, Aguascalientes, México) se presentó un CODI (contenido digital) para el aprendizaje de la pronunciación de las vocales en inglés. La idea surge de un deseo por didactizar la fonética a través de esquemas explicativos mínimos de pronunciación para sentar las bases de una adecuada percepción de las formas fonéticas de textos. Se produjo un CODI con el propósito de que el usuario llegue a 
JORGE LANDAVERDE Y TREJO

MODELO DIDÁCTICO MEDIACIONAL PARA PROMOVER EL APRENDIZAJE DE SEGUNDAS LENGUAS

ser capaz de leer los símbolos de pronunciación de las vocales, tal como se muestran en los diccionarios.

- En el SOMECE de 2004 (XX Simposio Internacional de Computación en la Educación, Puebla, México) se presentaron unos CODIs diseñados para un curso de comprensión de textos. La muestra es un paquete de cinco lecturas que se elaboró con apoyo del programa Macro Media Flash las cuales se estructuraron con base en un marco de trabajo estructurante 10. El paquete fue grabado en CDs para que los usuarios dispusieran de su uso en cualquier lugar y a cualquier hora.

- En el FEULE de 2005 (XIX Foro de Especialistas Universitarios en Lenguas Extranjeras, Colima, México) se presentaron componentes digitales tipo video-clips, para la elaboración de un texto interactivo. Se habló del por qué y del cómo diseñar CODIs. Se hizo una invitación a los colegas a incursionar en la producción de video con fines didácticos.

- En el SOMECE de 2005 (XXI Simposio Internacional de Computación en la Educación, Sonora, México) se habló del uso de los videoclips en el diseño de CODIs para promover la enseñanza de lenguas. Se relató una experiencia colaborativa en la que los aprendientes tomaron parte muy activa en la producción de videos.

- En este año de 2006 se piloteó un texto interactivo, resultado de la integración de componentes que poco a poco se han venido construyendo. A través de toda esta travesía ha estado presente la intención de promover la participación motivada y creativa de los aprendientes en el manejo de componentes que, finalmente, constituyen los nuevos contenidos: facilitadores de procesos mentales creativos y transformadores. En este sentido la adquisición/aprendizaje de segundas lenguas no es un fin en sí, sino una actividad mediadora de interrelaciones, intercomunicación, inter-reflexiones de diferentes niveles espaciales.

\section{b) Varias reacciones}

La reacción que nos ha importado más es la de los usuarios de los CODIs. Aún cuando la intencionalidad subyacente al diseñar y producir los CODIs era que pudiesen favorecer la autonomía en el aprendizaje, hemos caído en cuenta de que todavía es necesaria una guía y un acompañamiento del profesor. Aquí juega una parte muy importante la cuestión afectiva y las relaciones de autoridad. Al hablar 
JoRge LaNDAVERDE Y TREJO

MODELO DIDÁCTICO MEDIACIONAL PARA PROMOVER EL APRENDIZAJE DE SEGUNDAS LENGUAS

de cuestiones afectivas no se está haciendo referencia a desviaciones inmaduras, sino a un respeto (atención avisada) y un acompañamiento a la persona (planeación previsora de actividades para desarrollo de habilidades y estrategias); en cuanto a las relaciones de autoridad, éstas se refieren a la excentricidad de la influencia docente y al involucramiento creciente de los aprendientes en la misma elaboración y manipulación de los contenidos digitales, puesto que la autoridad emana del conocimiento que se comparte.

En general se ha observado que los aprendientes han mostrado mayor participación, renovada motivación y mejor desempeño en el aprendizaje del inglés.

\section{Posible generalización}

a) Se tiene la certeza de que los CODIs (Contenidos Digitales) que se están elaborando, además de ser utilizados como material complementario en las sesiones de clase de la educación formal, con cierta reestructuración pudieran ser utilizados en los procesos de aprendizaje con modalidades mixta y abierta para satisfacer la demanda de capacitación para adultos, actualmente poco atendida como se observa en la gráfica que a continuación se muestra:

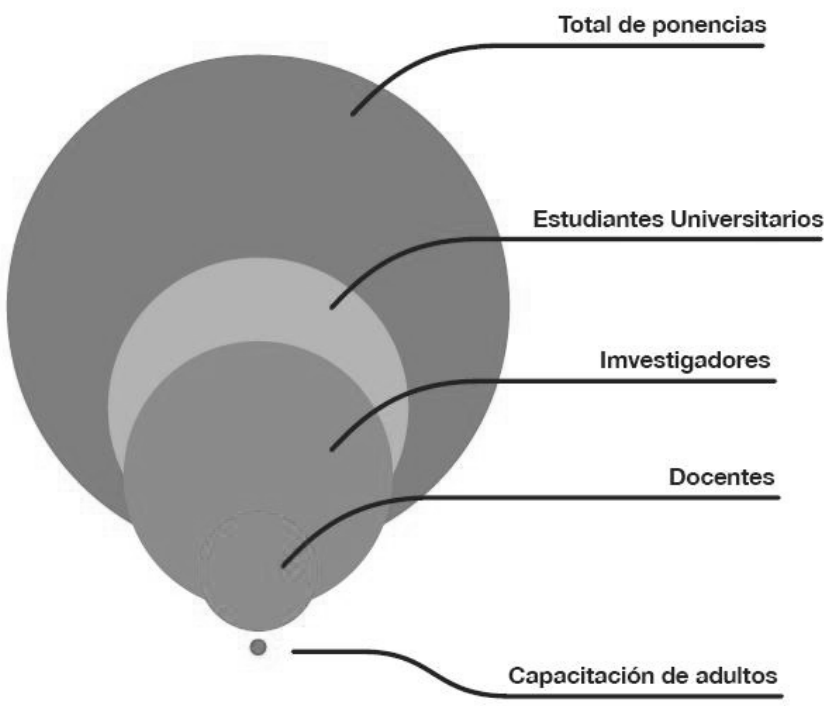

Gráfica de la cantidad de ponencias del XIX FEULE, según temática 
JORGE LANDAVERDE Y TREJO

MODELO DIDÁCTICO MEDIACIONAL PARA PROMOVER EL APRENDIZAJE DE SEGUNDAS LENGUAS

b) Los Marcos de Trabajo diseñados y aplicados en los CODIs producidos, los cuales pueden servir de modelo para implementar un banco de CODIs que a su vez puedan ser componentes de CODIs más complejos, si éstos se llegan a clasificar y almacenar en bancos repositorios, para un reuso sin fines lucrativos.

c) En el pasado ha habido intentos de elaboración de secuencias de contenidos que acompañen al aprendiente en sus procesos de lexicalizaciónsintaxización-relexicalización. Se sigue explorando y hace falta un rastreo, un registro y una organización de aquellas secuencias que puedan brindar un apoyo sustancial para acceder al dominio de segundas lenguas.

\section{NOTAS}

$1 \quad$ A partir de 1986 se ha venido celebrando el Foro de Especialistas Universitarios en Lenguas Extranjeras donde investigadores y docentes en el campo de la lingüística comparten sus hallazgos y avances de investigación. El XIX FEULE, del que aquí se hace referencia, se celebró del 7 al 10 de marzo de 2005, en la ciudad de Colima, México.

2 El autor Rolf Arnold (2005) desarrolla toda una teoría de la Andragogía en su libro Pedagogía de la formación de adultos, el cual tuve la fortuna de consultar vía Internet.

3 La autora Concepción Barrón Tirado, investigadora del CESU-UNAM, actualmente coordinadora del Posgrado en Pedagogía de la Facultad de Filosofía y Letras de la Universidad Nacional Autónoma de México, enmarca la educación basada en competencias dentro del contexto de las políticas educativas del estado mexicano, mediante un análisis histórico-crítico, en el capítulo sobre "La educación basada en competencias en el marco de los procesos de globalización”, del Número 91 de: Pensamiento Universitario: Formación en competencias y certificación profesional, coordinado por Valle Flores, María de los Ángeles, CESU-UNAM 2002.

4 Dentro del contexto de Virtual Educa 2005, la autora María Elena Chan Núñez hizo la presentación de su libro intitulado Modelo mediacional para el diseño educativo en entornos digitales, en el cual nos presenta un modelo paradigmático que trasciende la controversia en torno a las competencias laborales.

5 Peter Skehan en su libro: A Cognitive Approach to Language Learning (2001), analiza los diferentes modelos explicativos de los procesos de adquisición y aprendizaje de segundas lenguas, concluyendo con su propuesta teórica del sistema dual, la cual combina los sistemas basados en modelos con los basados en reglas. También señala la importancia de la distinción entre los procesos de adquisición de la lengua materna y los procesos de adquisición y aprendizaje de otras lenguas.

6 Para una profundización en este tema consultar el artículo escrito por Saniya Majmutova intitulado “Actividades en el salón de clase para la enseñanza explícita de la gramática” compilado en Lenguas y Diseño. Puntos de encuentro, por Gilbón, Dulce Ma et al. 2005, pp. 117-128. 
JORGE LANDAVERDE Y TREJO

MODELO DIDÁCTICO MEDIACIONAL PARA PROMOVER EL APRENDIZAJE DE SEGUNDAS LENGUAS

7 Para una comprensión mayor acerca de la diferencia entre los procesos de adquisición y aprendizaje podemos consultar el libro de Da Silva y Signoret intitulado Temas sobre la adquisición de una segunda lengua (2005) a partir de la distinción hecha por Stephen Krashen en cuanto que "adquisición describe un proceso de desarrollo similar al de los niños cuando desarrollan su L1... en oposición, como lo observamos tradicionalmente en las clases de idiomas, el aprendizaje es un proceso de desarrollo consciente de la lengua." (Da silva y Signoret 2004:55).

$8 \quad$ ¿Cuál es la lente que nos permite una mejor apreciación de la realidad? “...precisamente en función de las huellas inconscientes que dejan esos recuerdos, me decía a mí mismo, o más bien le decía a esa parte de mí que era Edgar Morín: ¿En qué consiste ese pensamiento de lo complejo si las operaciones de pensamiento que están a nuestra disposición son fabricadas por una primera lente de reducción, una segunda lente de aislamiento y una tercera de generalización?”, Coloquio de Cerisy en torno a Edgar Morín, coordinado por Daniel Bougnoux, et al. (2005).

9 El modelo educativo excéntrico propone el desplazamiento del profesor fuera del centro del acto educativo lo cual no significa restarle importancia. Requiere el desarrollo de competencias mediacionales para propiciar múltiples interacciones. El docente ya no es un controlador de recursos sino un facilitador de aprendizaje permitiendo que los aprendientes construyan significados a través de su libre interacción con entornos interactivos previamente dispuestos por el docente.

10 En relación a desarrollo de materiales, Brian Tomlinson escribió un libro intitulado

Materials Development in Language Teaching, en 2003.

\section{REFERENCIAS BIBLIOGRÁFICAS}

Barrón Tirado, Concepción, et al. (2002). $L a$ educación basada en competencias en el marco de los procesos de globalización. Formación en competencias y certificación profesional. México, D. F.: CESU-UNAM, 17-44.

Bougnoux, Daniel, et al. (2005). En torno a Edgar Morin. Argumentos para un método. Xalapa Veracruz: Universidad Veracruzana.

Chan Núñez, María Elena. (2004). Modelo mediacional para el diseño educativo en entornos digitales. Guadalajara: Universidad de Guadalajara.

Da Silva Gomes, Helena Ma, et al. (2005). Temas sobre la adquisición de una segunda lengua. México, D. F.: Editorial Trillas.

Gilbón, Dulce Ma. et al. (2005). Lenguas y Diseño. Puntos de encuentro. Líneas de investigación en el departamento de lingüística aplicada. México, D. F.: CELE-UNAM.

Rolf, Arnold (2005). Pedagogía de la formación de adultos. Internet. [en línea]. Disponible en: http://www.ilo. org/public/spanish/region/ampro/ cinterfor/public/arnold p/index.htm [consulta 2005, 12 de septiembre].

Ruiz-Velasco Sánchez (2005). Elementos para orientar el uso y la producción de contenidos digitales con certidumbre 
JORGE LANDAVERDE Y TREJO

MODELO DIDÁCTICO MEDIACIONAL PARA PROMOVER EL APRENDIZAJE DE SEGUNDAS LENGUAS

y calidad. Notas para el Seminario Especializado en Nuevas Tecnologías en el campo de la Educación. Diseño de entornos virtuales de aprendizaje. México, D.F.: CESU-UNAM.

Skehan, P. (2001). A Cognitive Approach to Language Learning. Hong Kong: Oxford
University Press.

Soto González, Mario (1999). Edgar Morín, Complejidad y Sujeto Humano. Tesis doctorado FFLUniversidad deValladolid. [en línea]. Disponible en: http://www. infoamerica.org/teoria/morin.htm [consulta 2005, 14 de septiembre].

\section{PALABRAS CLAVE}

Didactización, Mediación, Contenidos Digitalizados, Pluriculturalismo, Modalidad Mixta de Aprendizaje

\section{KEY WORDS}

Didactization, Mediation, Digital Contents, Pluriculturalism, Blended learning modality

\section{PERFIL ACADÉMICO DEL AUTOR}

Licenciatura en la enseñanza de las Matemáticas; Maestría en Ciencias de la Educación; Doctorado en Pedagogía. ; Certificado en enseñanza del inglés; Diplomado en la enseñanza de la Historia; Diplomado en Francés Básico; Diplomado en el uso de la computación en la Educación; Especialización en Coreano; Estudios en el ELS de Boston, Mass; ha participado en convenciones Mextesol desde 1984; ponente en múltiples foros. Coordinador de diversidad de diplomados; coordinador del área de idiomas; coordinador del área de investigación en FCAUAQ; Asociado a la Sociedad Mexicana de la Computación en la Educación, SOMECE desde 2003. Actualmente maestro e investigador de tiempo completo en la Universidad Autónoma de Querétaro.

Dirección postal: $\quad$ Calle Mariano Reyes 18; Colonia Centro; Santiago de Querétaro, Qro. CP 760oo; México. diplojlt@yahoo.com.mx; landatj23@hotmail.com

Fecha de entrega: 15.10.06

Fecha de aceptación: 19.12 .06 
JORGE LANDAVERDE Y TREJO

MODELO DIDÁCTICO MEDIACIONAL PARA PROMOVER EL APRENDIZAJE DE SEGUNDAS LENGUAS

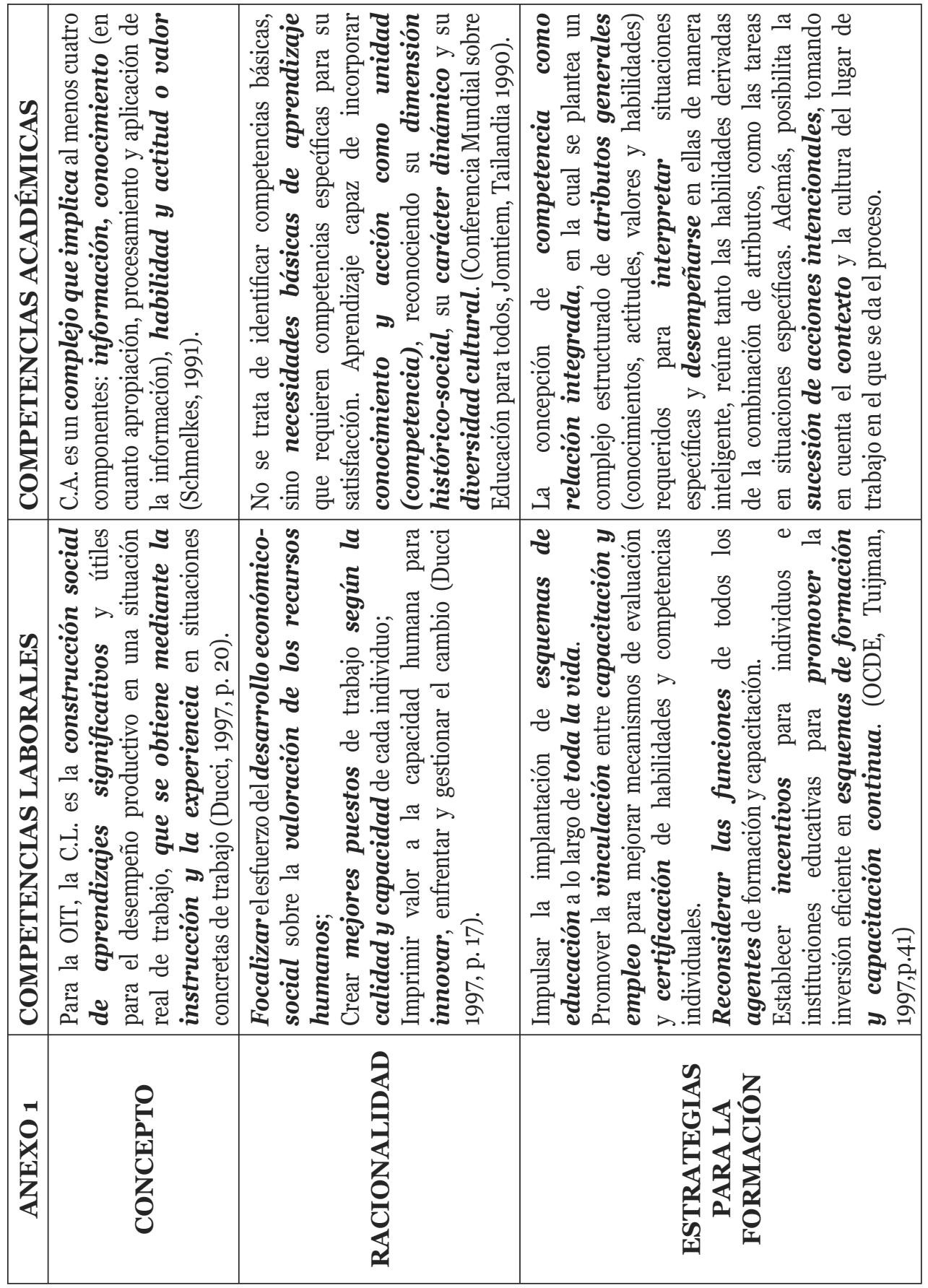

AIESAD

RIED v. 8: 1 y 2, 2005, pp 67-82 
JORGE LANDAVERDE Y TREJO

MODELO DIDÁCTICO MEDIACIONAL PARA PROMOVER EL APRENDIZAJE DE SEGUNDAS LENGUAS

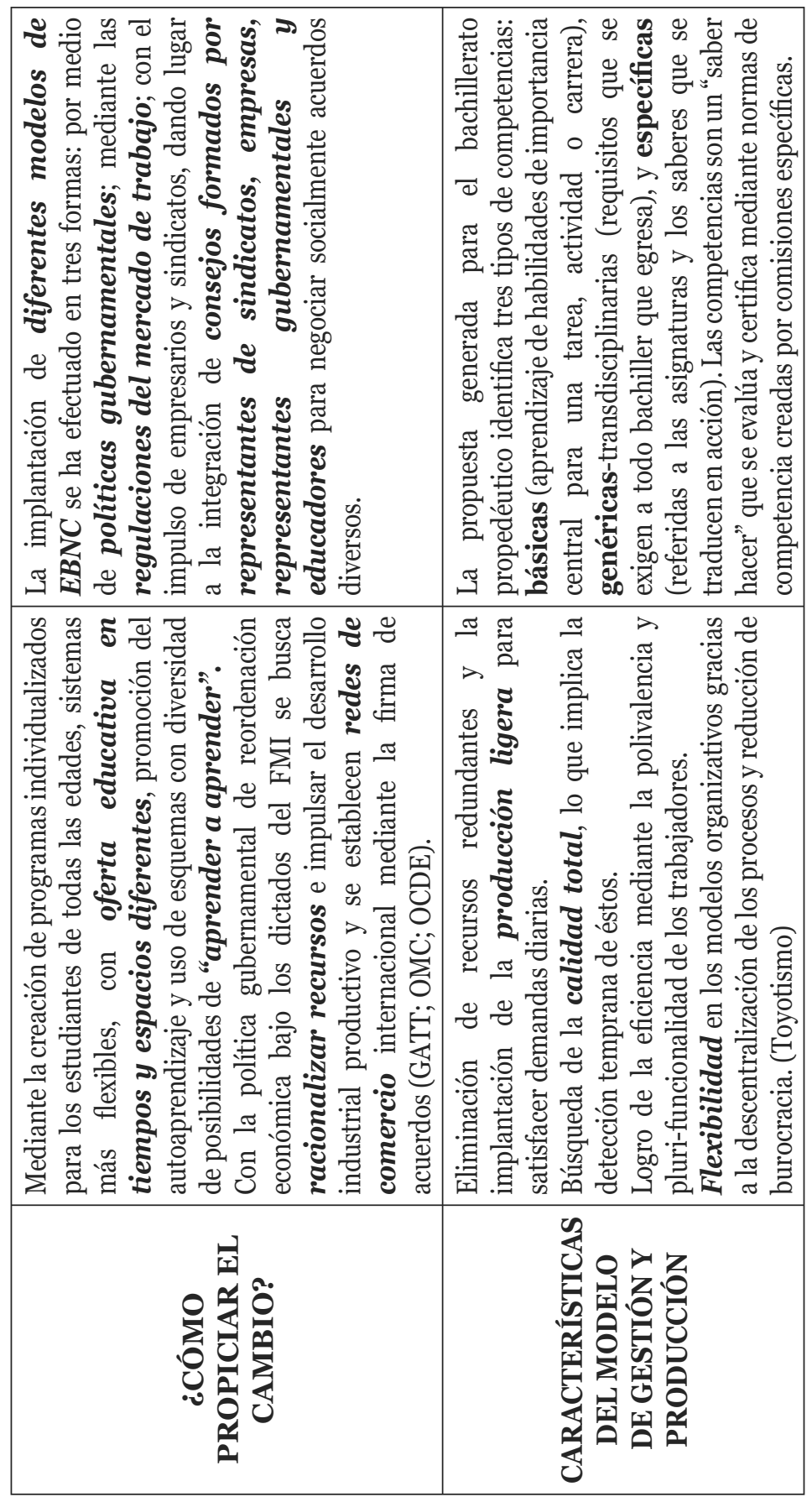

80 RIED v. 8: 1 y 2, 2005, pp 67-82

AIESAD 
JORGE LANDAVERDE Y TREJO

MODELO DIDÁCTICO MEDIACIONAL PARA PROMOVER EL APRENDIZAJE DE SEGUNDAS LENGUAS

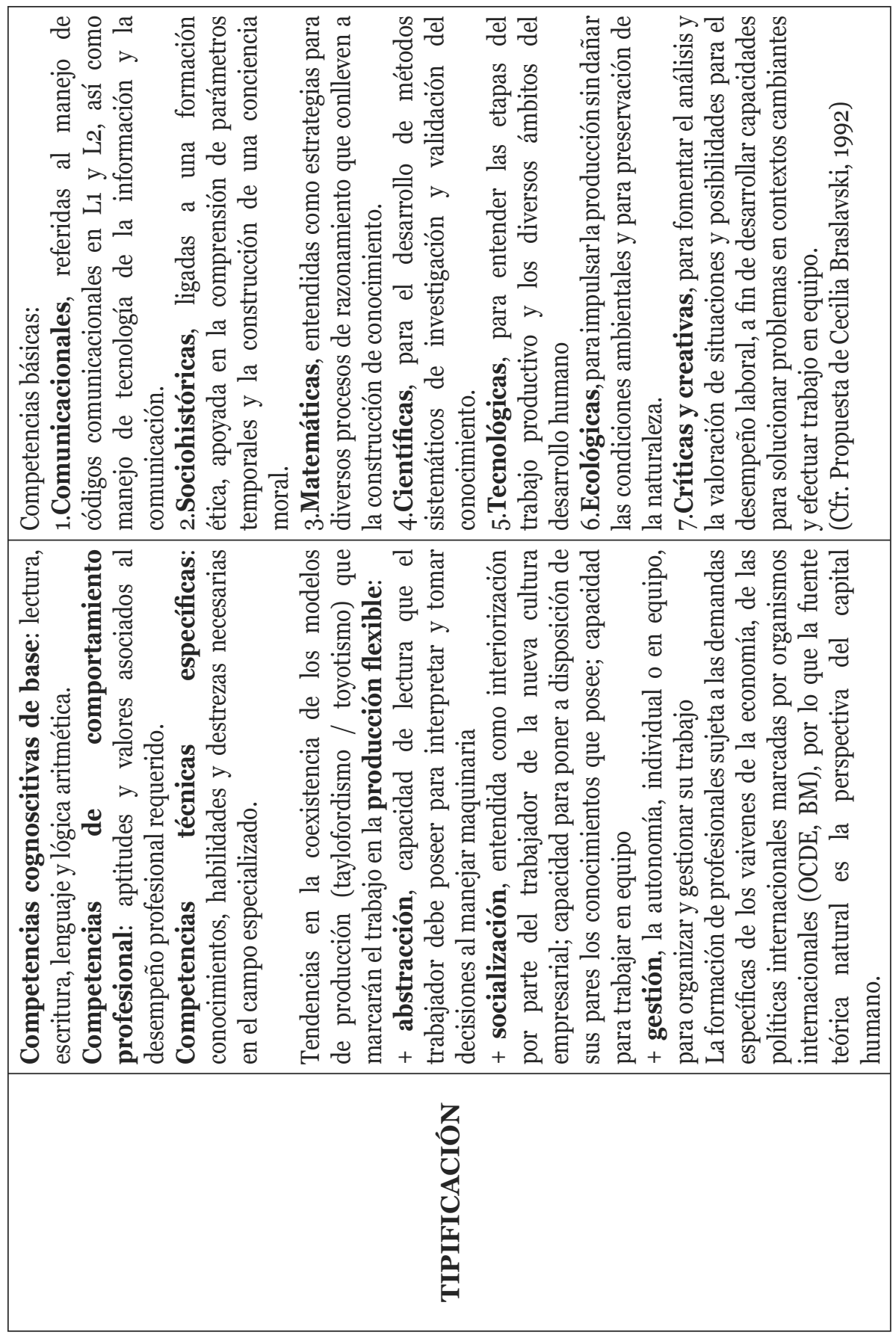

AIESAD

RIED v. 8: 1 y 2, 2005, pp 67-82 
JORGE LANDAVERDE Y TREJO

MODELO DIDÁCTICO MEDIACIONAL PARA PROMOVER EL APRENDIZAJE DE SEGUNDAS LENGUAS

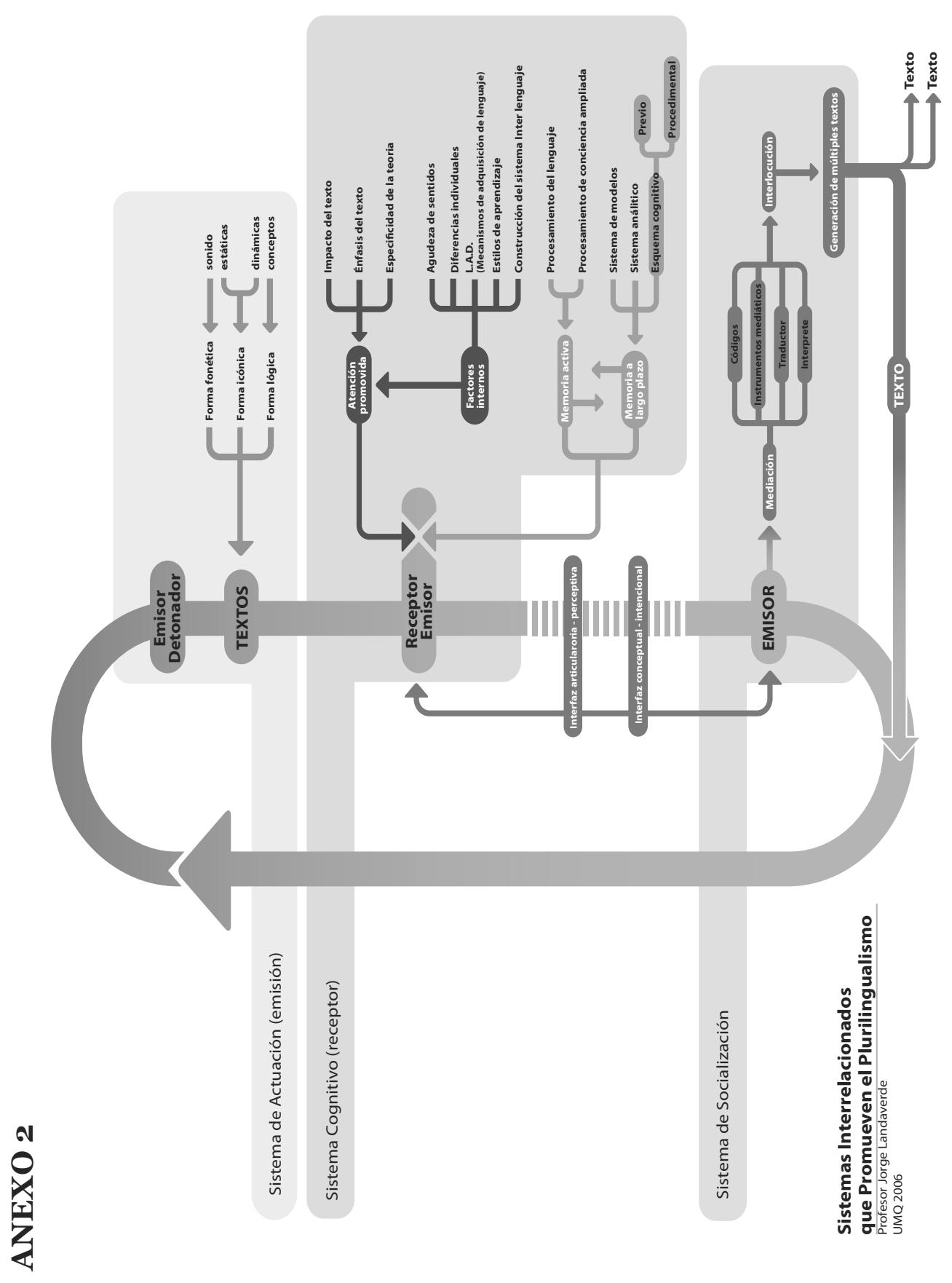

82 RIED v. 8: 1 y 2, 2005, pp 67-82 